\title{
Beam Transport Design for a Recirculating-Linac FEL Driver
}

D. Neuffer," D. Douglas, Z. Li," A. Garren, "“"M. Comacchia

"CEBAF, 12000 Jefferson Avenue, Newport News, VA 23606,

"SLAC, Stanford University, Stanford, CA 94305,

.... Lawrence Berkeley Laboratory, Berkeley, CA 94720,

-..'SSRL, Stanford University, Stanford, CA 94305

Contibuted Paper We1-25

RECEIVED

JUL 251996

OSTI

Corresponding author: .

David Neuffer MS 16A

Continuous Electron Beam Accelerator Facility

Newport News VA 23606 USA

Phone: 804-249-7613

E-mail: neuffer@cebaf.gov

\section{Abstract}

The beam transport system for the CEBAF Industrial FEL includes a two-pass transport of the beam with acceleration from injector to wiggler, followed by energy recovery transport from wiggler to dump. From that context, we discuss the general problem of multi-pass energy-recovery beam transport for FELs. Tuneable, nearly-isochronous, large-momentum-acceptance transport systems are required. The entire transport must preserve beam quality, particularty in the acceleration transport to the wiggler, and have low losses throughout the entire system. Various possible designs are presented, and results of dynamic analyses are discussed. 


\section{Introduction}

The CEBAF Industrial FEL Demo [1] is planned as a prototype and test bed for future highpower, high-efficiency, industrial-use FELs. It requires tuneable beam energies up to $\sim 200 \mathrm{MeV}$, with beam powers of $\sim 1 \mathrm{MW}$ and peak currents of $\sim 200 \mathrm{~A}$. Future practical applications will require costeffectiveness and energy-efficiency, and we incorporate into the Demo design features with these goals: multipass acceleration, which reduces the accelerator structure hardware for minimal total construction costs, and energy-recovery deceleration, which reduces the input power demands of the system.

Table 1 summarizes the requirements of the FEL Demo driver accelerator. For these requirements we have designed the accelerator system shown in Figure 1 , and it consists of a $10 \mathrm{MeV}$ photocathode-based injector, a 96 MV CEBAF-style SRF linac, and a two-pass recirculation transport. This system accelerates the beam from the injector through two passes of the linac to $200 \mathrm{MeV}$. The beam is then compressed and matched into the FEL wiggler, where it loses energy and greatly increases its energy spread while producing coherent light. The resulting beam is decompressed, returned into the linac, decelerated for energy recovery through two passes to $\sim 10 \mathrm{MeV}$, and then transferred into the dump. This two-pass design is a minimal configuration needed to demonstrate both recirculation and energy recovery, key features essential for future cost and energy efficient FEL drivers.

In this paper we discuss the beam transport system for the Demo, which is itself a prototype for multi-pass energy-recovery transport systems, and addresses the major difficulties in such systems. For both passes of the Demo we require tuneable, nearly-isochronous, large-momentum-acceptance transport systems, with preservation of beam quality, and low-beam-loss (high acceptance) throughout the system. In the following section we discuss the transport design considerations. This is followed by 


\section{DISCLAIMER}

Portions of this document may be illegible in electronic image products. Images are produced from the best available original document. 

discussion of several lattice types which we have considered in developing the FEL driver system. The current design and its variations are presented, and directions for further improvements are discussed.

\section{Lattice Design Considerations}

In the present scenario, the beam is received from the injector with a relatively long bunch length. That bunch length is maintained through the first pass acceleration and $105 \mathrm{MeV}$ returm transport, which is isochronous $\left(M_{s 6}=0\right)$. In the second pass through the linac the beam is accelerated off-crest by $\phi_{z} \sim-15^{\circ}$, so that a position-dependent energy tilt is placed upon the beam:

$$
\Delta E(\phi)=e V_{R F}\left(\cos (\phi)-\cos \left(\phi_{2}\right)\right) \approx-e V_{R F} \sin \left(\phi_{2}\right)\left(\phi-\phi_{1}\right)
$$

The second arc transport (200 MeV) from linac to wiggler is nonisochronous, which means that position within the bunch changes, dependent on energy:

$$
\Delta \phi=M_{s 6}\left(\Delta E / E_{0}\right)(2 \pi / \lambda)
$$

and is designed for compression to a high-peak current at the wiggler. In the linearized limit, this implies: $e V_{R F} \sin \left(\phi_{z}\right) M_{s \sigma} E_{0}(2 \pi / \lambda) \approx 1$, or $M_{s 6} \approx-0.25 \mathrm{~m}$ at our parameters. The beam is also focussed to a small transverse spot size at the wiggier $\left(\beta_{\perp} \approx 1 \mathrm{~m}\right)$. Transport to this 3-D focus must be obtained over the full momentum spread of the beam $( - \pm 1 \mathrm{MeV})$ with minimal distortion.

In the wiggler the beam loses energy in the FEL interaction and greatly increases its energy spread, to as much as $\Delta \mathrm{E}= \pm 5 \mathrm{MeV}$. This beam bunch is lengthened by the nonisochronous return 
transport to the linac, which places a position-dependent energy tilt upon the beam, while increasing the bunch length. That tilt is removed in off-crest deceleration through the first deceleration pass, where $\Delta E$ is reduced to $< \pm 1 \mathrm{MeV}$. Transversely, the beam is defocussed for matching back into the linac. This return transport is the inverse of the bunching process and requires similar transport properties, with the important difference that the enlarged $\Delta \mathrm{E}$ must be accommodated. Following the (off-crest) first deceleration pass (third linac pass), the beam is retumed through the $105 \mathrm{MeV}$ return transport for a second (energy-recovery) deceleration (on crest) to $\mathrm{E} \sim 10 \mathrm{MeV}$, where the beam is dumped. A critical requirement in the deceleration transport is low-losses, which implies lowdistortion, high-acceptance thoughout the system.

Both passes require control of the chronicity $\mathrm{M}_{56}$, which is defined and evaluated to first order by:

$$
M_{s \sigma}=\frac{\partial z}{\partial(\Delta E / E)}=\int \eta(s) \frac{B(s)}{B \rho} d s
$$

where $z=\lambda \phi / 2 \pi$ is position within the bunch, $\eta$ is the dispersion $(\eta=\partial \times / \partial(\Delta E / E)$, where $x$ is the transverse particle position), $B$ is the bending field in the dipoles and $B \rho=p / e$ is the rigidity. This quantity is naturally several meters in a typical transport. In our case we desire it to be zero (for the $105 \mathrm{MeV}$ arc) or slightly negative $\left(\mathrm{M}_{56} \sim-0.25 \mathrm{~m}\right.$ ) for bunch compression in the $200 \mathrm{MeV}$ arc. In a bending arc, $\eta$ and $B$ are naturally positive, and a natural lattice would have an $M_{56}$ of several meters. To obtain the desired $\mathrm{M}_{36}$ the lattice must be perturbed, with the introduction of negative bends and/or negative $\eta$. 
In addition to $M_{s 6}$ control, the transports must be achromatic (matched to $\eta=d \eta / d s=0$ ). They must also be betatron-function matched to obtain the correct beam-size envelopes. This means that the overall first order transport for the $105 \mathrm{MeV}$ transport must be $\pm I$, where $I$ is a unity transport, and that a matched focus into and out of a small beam spot at the wiggler must be obtained in the 200 $\mathrm{MeV}$ tranport. These properties must be maintained over the energy spread requirements $(\Delta \mathrm{E} \sim$ $\pm 1 \mathrm{MeV}$ in most of the machine, but $\Delta \mathrm{E} \sim \pm 5 \mathrm{MeV}$ in the $200 \mathrm{MeV}$ return transport from the wiggler to linac.). Compression must be linear over that energy range; this sets limits on the nonlinear chronicity $T_{566}\left(2 T_{366}=\partial^{2} z / \partial(\Delta E / E)^{2}\right)$. These chromatic limitations typically require some degree of secondorder sextupole correction. Minimizing higher order distortion implies correction with relatively weak sextupoles, which implies judicious sextupole placement. The lattice must also fit within a compact footprint for cost control; we have set a $10 \mathrm{~m}$ width as a design guideline.

For the UV Demo FEL, we must design two retum transport arcs, and both the low-energy $(\sim 105 \mathrm{MeV})$ and high-energy $(-200 \mathrm{MeV})$ passes are prototypes for transport systems of general utility. The $105 \mathrm{MeV}$ transport is a "transparent" transfer line designed to image beam from the end of the linac to the beginning without changing the beam size or length, and without distortion. In contrast, the $200 \mathrm{MeV}$ line is designed to focus the beam both longitudinally and transversely into a compressed size in the wiggler and to decompress it on return from the wiggler. 


\section{Particular Solutions}

In this section we discuss some particular approaches and solutions to this transport problem which we have explored. In these studies the program DIMAD[3] was used for lattice specification and second-order optics, and TLIE particle tracking [4] was used to study higher-order effects.

The MTT-Bates recirculating linac [5] utilizes an arc design which meets our basic design specifications. The achromatic, isochronous transport line (see Fig. 2) consists of two half-chicanes separated by a main $180^{\circ}$ bend. Dispersion suppression is provided by the horizontal imaging properties of the main dipole; proper selection of the dipole bend radii, bend angles and separation of the dipoles also provides $\mathrm{M}_{\$ 6}$ control. In particular the negative bend in the second chicane dipole, where $\eta$ is relatively large, coupled with a small $\eta$ in the $180^{\circ}$ bend permits $M_{56} \sim 0$. Fine tuning of $M_{\$ 6}$ can be achieved by introducing a pair of quadrupole doublet trims between the half-chicanes and the main dipoles. Chromatic aberrations, including $T_{366,}$ are suppressed by a judicious choice of gross system parameters, and through use of two families of chromatic correction sextupoles.

The Bates recirculator has a full momentum acceptance of $6 \%$; this is adequate for many FEL driver applications. We have generated generally-useful designs based on it, but we have found the following limitations. Designs with acceptable chromatic behavior for the $200 \mathrm{MeV}$ arc tend to be somewhat large transversely (greater than our $10 \mathrm{~m}$ desired width goal). Narrower designs tended to have a vertical chromatic aberration generating excessive $\partial \beta / \partial(\Delta E / E)$, and correction of this is difficult because the simple layout provides few distinct locations for correction sextupoles. Finally, largeenergy acceptance designs had large dispersions $(\eta)$ of several meters in some locations, requiring undesirably large-aperture magnets. 
In the FEL design effort we have invented an extension of the Bates arc, which is designed to overcome these limitations, and which is nicknamed the "Virginia Reel"(see Fig. 2). Like the Bates arc, dispersion and chronicity control are obtained by the dipole geometry rather than through quadrupole focussing. Unlike the Bates arc, the orbit geometry is managed in a gentler and more periodic manner. Specifically, rather than a single pair of chicane dipoles leading to and from a single main dipole, the Virginia Reel has a sequence of chicanes ( 4 dipoles) leading to/from a four-cell achromatic FODO arc, whch has a $360^{\circ}$ betatron phase advance. The use of multiple chicanes reduces the maximum $\eta$ to $\sim 1 \mathrm{~m}$, and reduces the chicane bend angles and radii; the chromatic aberrations are therefore reduced to manageable levels. As in the Bates case, $\eta$ and $B$ are of opposite signs in the chicane dipoles and this counteracts the naturally positive $\mathrm{M}_{56}$ of the FODO arc, permitting tuneable Ms6.

The use of a FODO arc, with sextupole placement within the arcs, provides opportunities for use of second-order achromatic symmetries in the implementation of chromatic correction schemes. The nonlinear lattice is therefore more easily managed than in other cases. The principal deficiency in the Virginia Rel is the relatively large space required to accommodate the multiple chicanes, and the subsequently large arc footprint. Another disadvantage is the relatively large number of magnetic elements.

A more compact geometry for a return arc can be obtained simply by a string of four FODO cells (see Fig. 2), each consisting of two quads and two dipoles, which can be obtained by removing the chicanes in the Virginia Reel. With $90^{\circ}$ betatron phase advance per cell, this would be a natural achromat, but would have a large positive $\mathrm{M}_{\$ 6}$. However, the quad strengths can be symmetrically perturbed so as to create a dispersion wave, with relatively large negative values in some of the dipoles, 
and the perturbation can obtain an achromat with a small tuneable chronicity $\left(M_{s 6} \approx 0\right)$. Thus, we have designed a four-cell "Perturbed FODO" lattice for the FEL. However, with only four cells and relatively tight bending, the $\eta$ perturbation produces a very large betatron-function wave (beam-size oscillation). Also the sextupole correction possibilities are limited, and the overall chromatic behavior for large $\triangle \mathrm{E} / \mathrm{E}$ was somewhat disappointing.

A compact, nearty-isochronous lattice can also be obtained using a "reverse-bend"-type lattice, originally developed by Amiry and Pellegrini. [6] This lattice consists of achromatic cells, each of which consists of a large positive bend, a set of matching quads (4 in initial designs), and a negative bend, followed by a mirror image set of matching quads and positive bend (see Fig. 2). The combination of small dispersion $(\eta)$ at large-angle bends and large (tuneable) $\eta$ at small negative-bends leads to nearty zero but negative $M_{56}$. For the FEL, an are consisting of two such cells was designed, with positive bends of $48.5^{\circ}$ and reverse bends of $7^{\circ}$, and found to have adequate dynamic behavior and to fit within a reasonably compact footprint.

A disadvantage of this configuration is the relatively large number of individual elements, which are relatively tightly packed within the present geometry, and it only accommodates negative $M_{56 .}$ A variation on this two-cell geometry with broader $\mathrm{M}_{56}$ tuneability has been designed in which the reverse bends are removed, and the quads perturbed from the two-cell symmetry to obtain negative (tuneable) dispersion at the interior dipoles of the two-cell geometry. With this perturbation, both positive and negative $\mathrm{M}_{56}$ can be obtained. This solution is more than adequate for the chromatic requirements of the transport to the wiggler $(\Delta E=1 \mathrm{MeV})$ over the full $\mathrm{M}_{56}$ range. 


\section{Current Design and Discussion}

In Figure 1 we display the current FEL accelerator design, as presented in the 1995 Conceptual design report.[2] The complete design includes the injector, $95 \mathrm{MeV}$ linac, and the two-pass transport for acceleration to the wiggler and energy-recovery deceleration to the beam dump. Injection, multipass beam combination, separation and extraction are managed in tuneable dipole chicanes, which allow independent energy adjustment in each line.

The present design uses Bates-type arcs for the 105-MeV transport and a reverse-bend arc as the basis for the $200 \mathrm{MeV}$ line. The $105 \mathrm{MeV}$ line consists of arcs with a $180^{\circ}$ central bend and two half-chicanes with plus and minus $20^{\circ}$ bends, and these are arranged to obtain isochronous and achromatic motion. The straight section contains three quad triplets, with the overall $x$-y transport fitted to a -I matrix.

The $200-\mathrm{MeV}$ transport is the outer loop of Figure 1 . The $200-\mathrm{MeV}$ line carries the tham around the high-energy arc from the linac to the wiggles and returns it for energy recovery. This transport is non-isochronous to enable bunching and debunching, and it must accept a large energy spread, particularly in the beam exiting the wiggler, and have broad tuneability in transverse and longitudinal focusing. The present design meets these requirements. The 200-MeV arcs are based on a two-cell reverse bend configuration. The arc quads can be varied to change the chronicity $\mathrm{M}_{56}$ by \pm 0.05 by minor retunings, and to obtain $\mathrm{M}_{s 6}=0 \pm 0.30$ with larger changes. The complete lattice includes the beam-separation and path-length adjustment chicanes as well as matching transports into both $U V$ and $I R$ wigglers. Figure 1 includes the bypass transport into the $\mathbb{R}$ wiggler, which is parallel to the UV wiggler. 
Note that in figure 1 the reverse-bends have been removed in the transport to the wiggler. This enables broader chronicity tuning, over $M_{56}= \pm 0.3$. From if considerations it has been shown that opposite signs of $\mathrm{M}_{36}$ in the transports to and from the wiggler, respectively, may be more stable [7], and this modification permits this variant operation.

Further development and modification of this lattice is expected as the project progresses toward construction. While the $105 \mathrm{MeV}$ line has more than adequate linearity for on-crest accelerated and decelerated beam $(\Delta \mathrm{E} \sim \pm 1 \mathrm{MeV})$, variant if operation [ 7$]$ would require off-crest operation with a larger energy aperture $(\Delta \mathrm{E} \sim \pm 3 \mathrm{MeV})$. Our studies indicate that an enlarged energy aperture could be obtained with weaker bending, and future studies will explore this variation. The $200 \mathrm{MeV}$ arcs appear to have more elements than necessary. The four quads per reverse-bend half-cell should be adequately $\because:$ replaced by triplets, and the number of matching quads could be reduced. The lattice is also being adapted for optimal magnet design constraints. Reduced and simplified configurations will be explored. Configurations greatly different trom the present options should also be developed and studied. Collective effects studies, such as space-charge and coherent radiation, as well as rf stability considerations may also modify the transport requirements and demand further design variations.

\section{Acknowledgments}

This research was supported by the Virginia Center for Innovative Technology and DOE Contract \#DE-AC05-84ER40150. 


\section{References}

1. G. R. Neil, S. V. Benson, H. F. Dylla, and H. Liu, Nucl Inst. and Meth. A358,159 (1995).

2. D. V. Neuffer, et al., "Accelerator Design for the High-Power Industrial FEL", Proc. 1995 Particle Accelerator Conf. ,Dallas TX (1995)

3. R. V. Servranckx, K. L. Brown, L. Schachinger, and D. Douglas, SLAC Report 285, UC-28A (1985).

4. J. van Zejits and F. Neri, "The Arbitrary Order Design Code TLIE", (unpublished) 1994.

5. J. Flanz, G. Franklin, S. Kowalski, and C. P. Sargent, MIT-Bates Laboratory "Recirculator Status Reports," unpublished (1980); described in R. Rd, Recirculating Electron Accelerators, Harwood Academic Publishers, NewYork, 1984, pp. 107-109 and 155-8.

6. A. Amiry and C. Pellegrini, "Design of a Quasi-isochronous Light Source," Proc. Workshop on Fourth Generation Light Sources, SSRL92/02 (1992).

7. L. Merminga, J. Bisognano, and J. Delayen, "Energy Stability in Recirculating, Energy-Recovering Linacs", Proc. 1995 FEL Conference, New York (1995). 
Figure 1. Overview of FEL Driver Accelerator, showing $10 \mathrm{MeV}$ injector, $96 \mathrm{MV}$ linac, first-pass recirculation transport for $105 \mathrm{MeV}$ beam, and second-pass transport which takes $200 \mathrm{MeV}$ beam to the UV or IR FEL wiggler and returns it to the Linac for energy recovery.

Figure 2. Layout plots for return arcs of candidate lattices - Bates-type arc, Virginia reel, perturbed FODO, two-cell reverse-bend. 
Table 1 - FEL Accelerator Parameters

Electron Energy

Accelerator type

E $200 \quad \mathrm{MeV}$

two-pass SRF recirculator,

with two-pass energy recovery

RF frequency

$f=\omega / 2 \pi \quad 1.4997 \mathrm{Ghz}$

RF voltage

$V_{\text {f }}$

$96.3 \mathrm{MV}$

RF phases (Passes 1, 2, 3, 4)

Charge/bunch

$\phi_{1}, \phi_{2}, \phi_{3}, \phi_{4} \quad 2.8^{\circ},-12.5^{\circ}, \pi+15.8^{\circ}, \pi+0.5^{\circ}$

Q $135 \quad \mathrm{pC}$

Transverse emittance

UV wiggler betatron amplitude $\varepsilon_{N} \quad 11 \quad \mathrm{~mm}$-mrad

$\beta_{\perp} \quad 1 \mathrm{~m}$

Transverse beam size at wiggler $\sigma=\left(\varepsilon_{N} \beta_{1} \gamma\right)^{1 / 2} \quad 0.17 \quad \mathrm{~mm}$

rms energy spread at wiggler $(\sigma)$

ms bunch length at wiggler

$\Delta E / E$

$0.15 \%$

$\Delta \phi_{\min }$

0.2 ps 


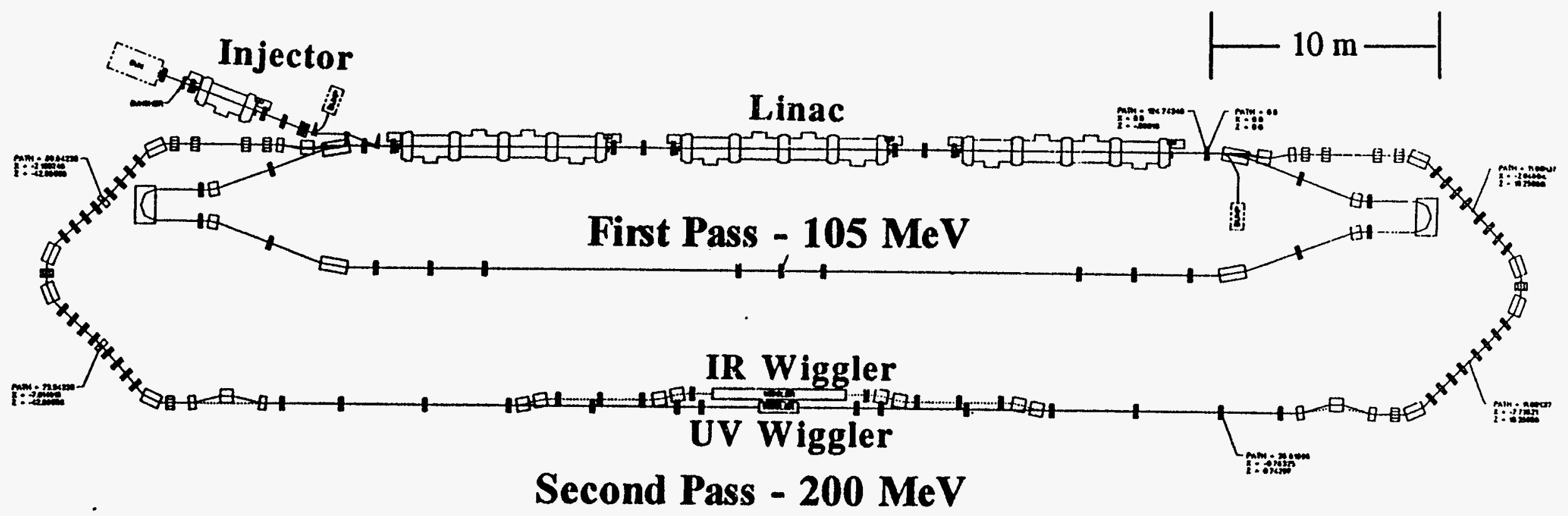




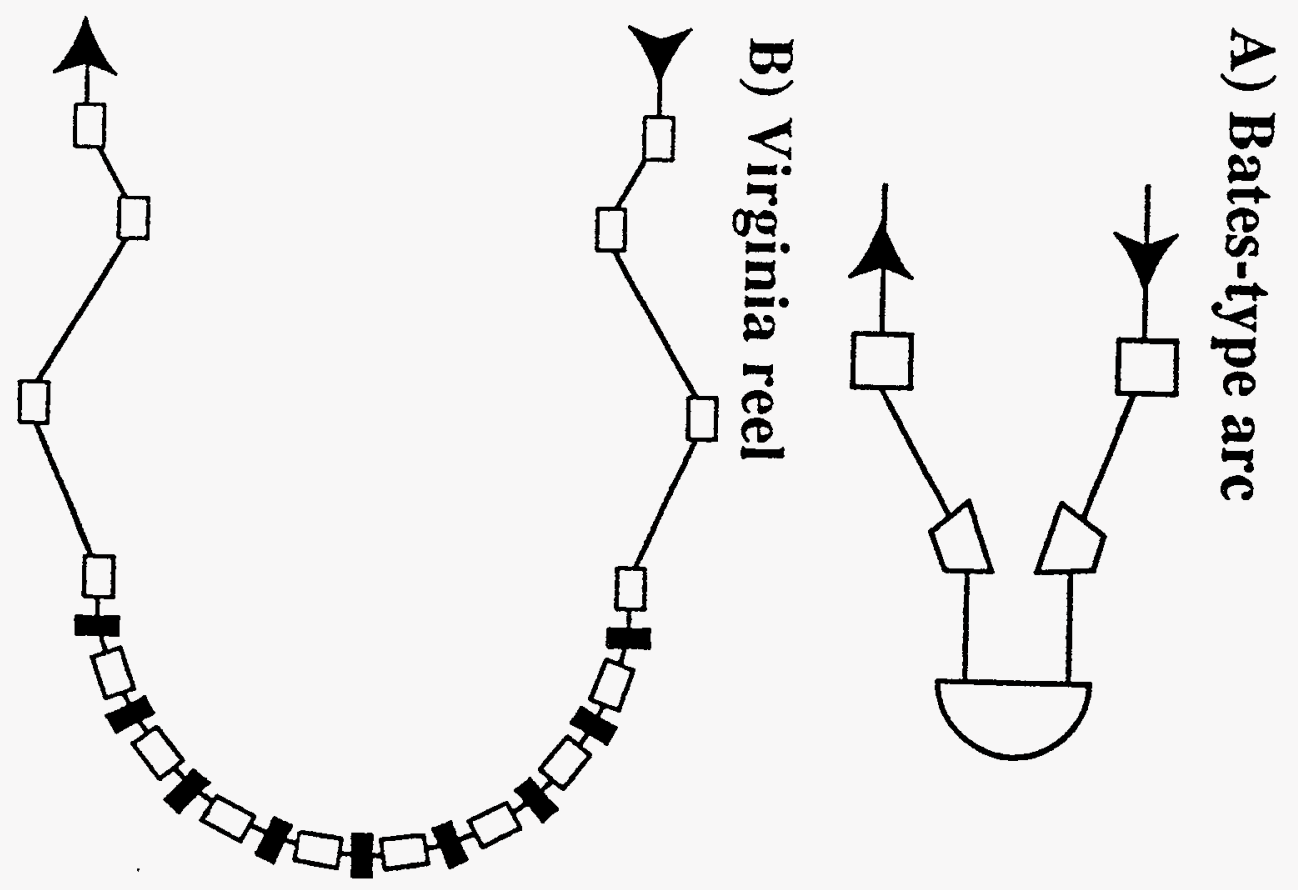

홀

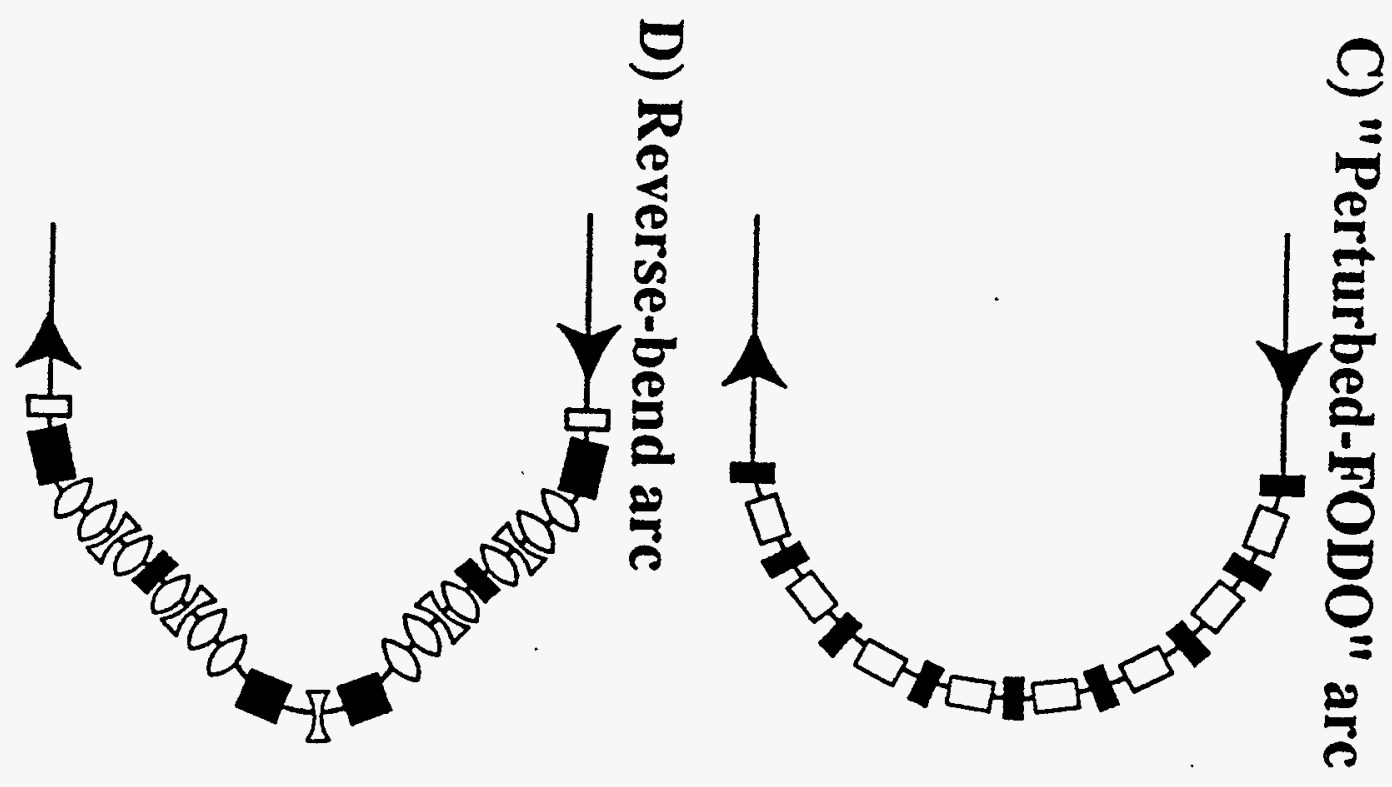

